debilitating disease. It also results in heavy use of medical services and frequent hospital admissions. Patients need information, self care skills, education and training to be able to self manage their condition. Following extensive consultation with local COPD patients and the respiratory multidisciplinary team (MDT), an individualised management plan was formulated and implemented into primary, intermediate and secondary care.

Aims The aim of the record is to increase the knowledge and control patients have of their disease and provide patients and carers with easily identifiable and accessible sources of support

Methods Fifty COPD patients completed the Bristol COPD Knowledge Questionnaire. The results highlighted many knowledge gaps, despite the mean length of time from diagnosis being $>5$ years. COPD patients and carers also attended a focus group, where the same knowledge gaps were highlighted. This group also provided advice on how the information should be presented and the type of language and terminology that should be used. A second focus group consisting of members of the respiratory MDT was then held and the final format for the record agreed.

Discussion The information found in the hand-held record is based on identified gaps in knowledge in the local COPD population and includes:

- What COPD is

- Generic COPD management

- Individual patient and carer management

- Goal setting

- Pharmacological management

- Exacerbation management

- End of life supportive care

- Telehealth

The hand-held record has been well received by patients and health care professionals alike. The document is a 'live' document and is being further developed. Patient and carer education groups have been run over a 4-week period.

Conclusion Developing and implementing the COPD hand-held record has highlighted the knowledge gaps in the identification, management and education needs not only in patients with COPD but in health care professionals. Working in an integrated manner with patients, carers and colleagues from primary, intermediate and secondary care has helped bridge the knowledge gaps and led to better outcomes for patients and health care professionals alike.

\section{P234 BLF AND BTS "READY FOR HOME" SURVEY OF THE EXPERIENCES OF PATIENTS ADMITTED TO HOSPITAL WITH COPD. PT2: THE DISCHARGE PROCESS}

doi:10.1136/thx.2010.151068.35

J E Scullion, S J Singh, M D L Morgan. University Hospitals of Leicester, Leicester, UK

Introduction and Objectives A secure discharge from hospital following an admission for COPD is an important reflection of the quality of hospital care. Information about the discharge process will help inform strategies to prevent unnecessary re-admissions.

Methods An 8-item questionnaire was sent to respiratory departments in all acute hospitals in the United Kingdom. Recipients were asked about their hospital's processes and the provision of information to patients at discharge.

Results 68 hospitals returned the questionnaire. There was coverage of hospitals from all regions of England as well as Scotland and Wales. The majority (69\%) of responses came from district general hospitals (DGHs). Catchment populations ranged from 100000-1 million. Respiratory departments varied in size from $<20$ beds $(10.3 \%)$ to $>60$ beds (11.8\%). Almost half the departments $(48.6 \%)$ have $>70$ COPD admissions per month. There was an early discharge service in many hospitals (71\%), with most being based in the hospital $(67 \%)$ vs the community. Only $52.9 \%$ patients were seen by a respiratory physician before discharge; the majority (69.1\%) were seen by a specialist nurse. Most hospitals (52.9\%) did not have a formal discharge check list or discharge pack (63.2\%). Many hospitals (69\%) give patients a copy of the discharge letter, and some refer patients directly to pulmonary rehabilitation (54\%) or smoking cessation services $(63 \%)$ on discharge. Follow-up appointments within primary or secondary care were not universally arranged and there was patchy coverage of educational advice to patients (range: $45 \%-75 \%$ ), especially on return to work or sexual relationships.

Conclusions Whilst admissions for exacerbations of COPD are common, preparation for discharge is unsatisfactory. Many patients do not see a specialist or have appropriate post-discharge advice or follow-up arrangements.

Funding The survey was supported by an educational grant from Nycomed.

\section{P235 BLF AND BTS "READY FOR HOME" SURVEY OF PATIENTS ADMITTED TO HOSPITAL WITH COPD: THE HOSPITAL EXPERIENCE}

doi:10.1136/thx.2010.151068.36

I Jarrold, N Eiser. British Lung Foundation, London, UK

Introduction and Objectives The patient experience is an important component of the quality of care. The objective of this survey was to explore the experiences of patients who were admitted to hospital with an exacerbation of Chronic Obstructive Pulmonary Disease (COPD).

Methods Patients were asked about their last hospital admission for COPD using a 14-item, self-administered questionnaire. They were approached through Breathe Easy groups, the British Lung Foundation website and a commercial market research panel

Results 307 COPD patients responded to the survey of whom $83 \%$ were aged $\geq 56$ years; $89 \%$ had established COPD (diagnosed $>12$ months), and $39 \%$ had been admitted to hospital within the last 12 months. Only 45\% were aware that "exacerbation" had been the cause of their admission. $56 \%$ had $\geq 24$ h notice of illness prior to admission and $43 \%$ initiated the admission themselves, without contacting a health care professional (HCP). Only 25\% patients felt 'very' confident that they were ready for their hospital discharge and $26 \%$ in their ability to cope at home. $34 \%$ patients felt very well informed about their COPD, the reasons for admission and their ability to identify early signs of an exacerbation. Only $37 \%$ were very reassured at receiving adequate support at home and only $24 \%$ were very positive about the future. $75 \%$ patients wanted more information regarding the reasons for their admission, $80 \%$ on treatment choices, $68 \%$ on COPD patient organisations, $78 \%$ on HPC support available at home and $69 \%$ on lifestyle, social and other coping problems. More contact from HCP support services and support via a telephone helpline were also requested $75 \%$ and $64 \%$, respectively). Despite this, $60 \%$ felt 'very' confident about how and when to take their medications.

Conclusions Hospital admission remains a significant risk to patients with established COPD, especially if they have had previous admissions. Data suggest that many patients feel unready for hospital discharge and are poorly prepared for the future management of their COPD. Hospital admission should be taken as an opportunity to provide patients with more information on COPD and available support services.

Funding The survey was supported by an educational grant from Nycomed. 